\title{
The long and winding road for overcoming resistance to hormone therapy in breast cancer
}

\author{
Shigehira Saji ${ }^{1}$
}

Published online: 28 May 2018

(c) The Japanese Breast Cancer Society 2018

Endocrine therapy is a main modality for hormone receptor (HR)-positive breast cancer. In the treatment of advanced or metastatic HR-positive breast cancer (ABC/MBC), endocrine therapy is generally used as sequential therapy, which uses a single drug one by one when the disease progresses.

Although this is due to consistent dependency on the estrogen receptor (ER) system in HR-positive breast cancer, in later periods of $\mathrm{ABC} / \mathrm{MBC}$ treatment, tumors gradually lose the response to this type of endocrine drugs. Many drugs have been developed to date, and recent progress has focused on molecular targeting agents combined with endocrine therapy for overcoming this issue of resistance to hormone therapy.

Several years ago, we postulated a schematic presentation of resistant mechanism to endocrine therapy in HR-positive breast cancer using a hybrid car model (Fig. 1) [1, 2]. In general, a hybrid car has two power sources - a gasoline engine and an electric motor. The gasoline engine and the electric motor are ER and growth factor receptors, respectively. Signaling pathways such as the phosphatidylinositol 3-kinase (PI3K)/Akt/mammalian target of rapamycin (mTOR) are transmission systems from the electric motor to the drive shaft, or gears from the motor to the engine to assist the engine. Downstream gene expression from ER is the transmission from the engine to the drive shaft. In this model, aromatase inhibitor works for deprivation of the gasoline, and selective estrogen receptor modulator (SERM) such as tamoxifen works as non-function gasoline. Because of this, the car (cancer) is unable to move. However, breast cancer is smart enough to start to use the motor to restart the car. In this situation, signaling pathway inhibitor should be

Shigehira Saji

ssaji@fmu.ac.jp

1 Department of Medical Oncology, School of Medicine, Fukushima Medical University, 1 Hikarigaoka, Fukushima City, Fukushima 960-1295, Japan useful for stopping the motor driven car, and for avoiding a restart by engine. Endocrine therapy should be accompanied with it.

In this special feature issue of Breast Cancer, three articles reported recent progress of this topic. Hanamura et al. described a potential endocrine resistance mechanism in breast cancer found via cell studies and animal models [3]. They established six types of aromatase inhibitor-resistant breast cancer cell lines, and showed us an important issue where a resistance mechanism varies in each cell and might be the mixture of several types of resistance mechanisms in "real world" patient's tumor. The research on this area has been conducted intensively through molecular biology experiments using long-term estrogen deprivation condition since 1990 [4]. Long-term cell culture in low or estradiol deprivation condition changes estrogen-dependent breast cancer to independent one. Comparing signaling pathways, receptor expression, and gene alternation between the original cell and the resistant cell is a useful method for exploring the acquired mechanisms in this process. In general, this type of experiments was done using a mass of long-term cultured cells. However, Hanamura et al. had tried to clone a single cell from a culture plate using a functional marker, and they divided the resistant mechanisms into six types. In our hybrid car model, these mechanisms could be explained as the use of motor power (type 1,2, and 3), use of other types of gasoline (type 4 and 5) and use of other types of engines (type 6).

Among these resistance mechanisms, the PI3K/Akt/ mTOR signaling pathway is the most well-known activated pathway, which has been the target of molecular target drugs such as mTOR inhibitor everolimus. Araki et al. has summarized the recent progress of this research and new clinically developed molecular target drugs [5]. Accumulating genetic studies have shown the important role of PI3K/Akt/ mTOR and cyclin-dependent kinase (CDK) 4/6/ retinoblastoma protein $(\mathrm{RB})$ pathways in cancer cell growth and 


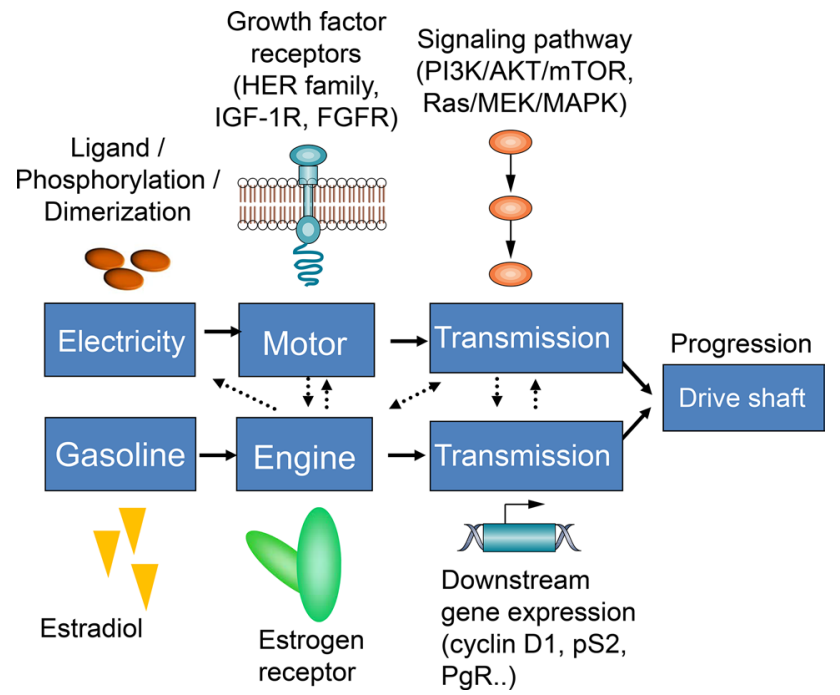

Fig. 1 Hybrid car model for understanding the resistance mechanism of hormone-dependent breast cancer. A hybrid car has two power sources; a gasoline engine and an electric motor. These are estrogen receptor (ER) and growth factor receptors such as HER family, IGF$1 \mathrm{R}$, and FGFR, respectively

survival. This is reflected by the relatively high frequency of the genetic alternation of PIK3CA (which encodes PI3K catalytic subunit- $\alpha$ ), $A K T 1$ (AKT) mutations, amplification of $C C N D 1$ (cyclin D1), loss of $C D K N 2 A$ (p16), and CDKN2C (p18) in HR-positive HER2-negative breast cancer.

Although several new drugs are waiting to be introduced in clinical settings, at this moment, CDK4/6 inhibitors are the leader of combination drugs used with endocrine therapy. Iwata has reported the history of CDK 4/6 inhibitor development and summarized the recent results of phase-III clinical trials [6]. The idea of CDK inhibitors as an anti-cancer drug has a long history, but it was not going well due to non-specific adverse events and a low specificity in targeting
CDK [7]. The development of CDK4- and CDK6-specific inhibitors makes it possible to use CDK inhibitors in cancer treatment. In our hybrid car model, the CDK4/6 inhibitor works as a stopping proximal part of transmission, drive shaft of the car, so that this agent effectively stops the car.

Based on the efforts of basic research in cell biology and translational research in clinical trials, it has gradually become clear that the resistance mechanism to endocrine therapy is a target of new molecular agents. We expect that more powerful agents will be available in clinical practice. However, breast cancer is very smart and tough and will develop new pathways to become resistant to new agents, so our efforts driving down the long and winding road will continue.

\section{References}

1. Saji S, Sato F, Ueno NT. Fuel, electricity, ER and HER2-a hybrid-car model of breast cancer. Nat Rev Clin Oncol. 2012. https://doi.org/10.1038/nrclinonc.2012.9-c1.

2. Saji S, Kimura-Tsuchiya R, Sasaki E. Molecular diagnostics for precision medicine in breast cancer treatment: what does the future hold? Breast Cancer. 2016;23(1):1-3.

3. Hanamura T, Hayashi SI. Overcoming aromatase inhibitor resistance in breast cancer: possible mechanisms and clinical applications. Breast Cancer 2017. https://doi.org/10.1007/s1228 2-017-0772-1.

4. Murphy CS, Meisner LF, Wu SQ, Jordan VC. Short- and longterm estrogen deprivation of T47D human breast cancer cells in culture. Eur J Cancer Clin Oncol. 1989;25(12):1777-88.

5. Araki K, Miyoshi Y. Mechanism of resistance to endocrine therapy in breast cancer: the important role of PI3K/Akt/mTOR in estrogen receptor-positive, HER2-negative breast cancer. Breast Cancer. 2017. https://doi.org/10.1007/s12282-017-0812-x.

6. Iwata $\mathrm{H}$. Clinical development of CDK4/6 inhibitor for breast cancer. Breast Cancer. 2018. https://doi.org/10.1007/s 1228 2-017-0827-3.

7. Shapiro GI. Cyclin-dependent kinase pathways as targets for cancer treatment. J Clin Oncol. 2006;24(11):1770-83. 